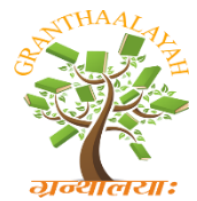

INTERNATIONAL JOURNAL OF RESEARCH GRANTHAALAYAH A knowledge Repository

Science

\title{
KINETIC STUDY OF THE ADSORPTION OF THE REMOVAL OF BROMO CRESOL PURPLE FROM AQUEOUS SOLUTIONS
}

\author{
Enas A. Almadani ${ }^{* 1}$, Hamad. M. Adress. Hasan ${ }^{2}$, Safwan. F. Kwakab ${ }^{3}$ \\ ${ }^{1,2,3}$ Chemical Department, Faculty of Science, Omar Al-Mukhtar University, Al Bayda
}

\begin{abstract}
This study was carried out to estimate the kinetic behavior of adsorption of bromo cresol purple from aqueous solution by using low cost of natural materials (sea grasses). The calculations of adsorption system were evaluated according to Langmuir adsorption and Freundlich isotherms. Also, the removal percentage of the studied dye was calculated. The results recorded that the values of $\mathrm{RL}$ were found to be less than (1) and slightly high than $(0)$ value $\approx 0.014$ indicating the favorable adsorption of the selected dyes on the sea grasses. On the other side the study recorded that the adsorption isotherms are harmony with the Freundlich concept of adsorption. According to the values which obtained from the isotherms in this study of bromo cresol purple. The adsorption process follows the first order reaction.
\end{abstract}

Keywords: Kinetic Study; Bromo Cresol Purple; Adsorption; Aqueous Solution; Sea grasses.

Cite This Article: Enas A. Almadani, Hamad. M. Adress. Hasan, and Safwan. F. Kwakab. (2019). "KINETIC STUDY OF THE ADSORPTION OF THE REMOVAL OF BROMO CRESOL PURPLE FROM AQUEOUS SOLUTIONS." International Journal of Research Granthaalayah, 7(12), 144-149. https://doi.org/10.29121/granthaalayah.v7.i12.2019.308.

\section{Introduction}

Adsorption became one of the most effective methods to remove color from aqueous solution (1). Despite the frequent use of adsorption in wastewater treatment systems, commercially available activated carbon remains an expensive material. Despite the frequent use of adsorption in wastewater treatment systems, commercially available activated carbon remains an expensive material. The two methods of processing adsorbents are physical and chemical methods. Physical method of treating adsorbent involves activation by heating in an oven (2). Chemical method of treating adsorbent involves activation by adding acid or adding some oxides. Since the addition of inorganic acids makes the method polluted and expensive, sometimes, combination of both methods may also be used (3). Cost is an important factor for comparing the feasibility of adsorbents in treating dyeing industry effluents. However, in any report, cost analysis is not stated and the expense of adsorbents varies depending on the method of processing and availability of source materials (4). In general, an adsorbent is said to be low cost if it requires little processing, abundant in nature with high adsorption capacity (5). For a compound to adsorb at a surface, a 
driving force must exist so that the compound will leave the aqueous phase. Examples of these include hydrophobic forces, electrostatic forces chemical attractions or vander Waals forces (6). Electrostatic forces drive adsorption when an ionic compound encounters an oppositely charged ionic adsorbent. Although these forces are a result of the charge of the compounds and their chemical make-up, adsorption due to electrostatic forces is classified as chemical adsorption (6). In physical adsorption, the energy (enthalpy) of adsorption is low (7). An example of chemical adsorption is when a compound chemically bonds to the surface to which it is adsorbing. Activated carbons often have chemical functional groups at the edges of the graphitic planes; therefore compounds can adsorb strongly via chemical adsorption these active sites (8). The activated carbon of sea grasses as low cost material to removal of some dyes including bromo cresol purple indicator was used, The aims of this study are using the sea grasses for the removal of the industrial organic dye ; bromo cresol purple from aqueous solutions. Then Using of some mathematical relationships by calculated the Langmuir, Freundlich isotherms and kinetics of adsorption.

\section{Materials and Methods}

\subsection{Preparation of Sea Grasses Carbon}

Sea grasses collected from coastline of Al-Gabal Al- Akhdar region. The sea grass samples were washed several times by distillated water then dried at overnight place for many days, and burned in the oven at $500^{\circ} \mathrm{C}$ for two hours.

\subsection{Preparation of Bromo Cresol Purple Solution}

A stock solution of the studied dye of $100 \mathrm{ppm}$ was prepared by dissolved the appropriate amount of dye in amount of water and made up to $100 \mathrm{ml}$ mark with deionized water. Different concentrations ranged between $10-50 \mathrm{mg} \mathrm{L}^{-1}$ of the dye were prepared from the stock solution. Deionized water was used for prepare all of the solutions and the reagent.

\subsection{Adsorption Studies}

The concentration of absorbed dye was obtained by comparing the recorded absorbance from a standard curve of the dye, Figure (1).

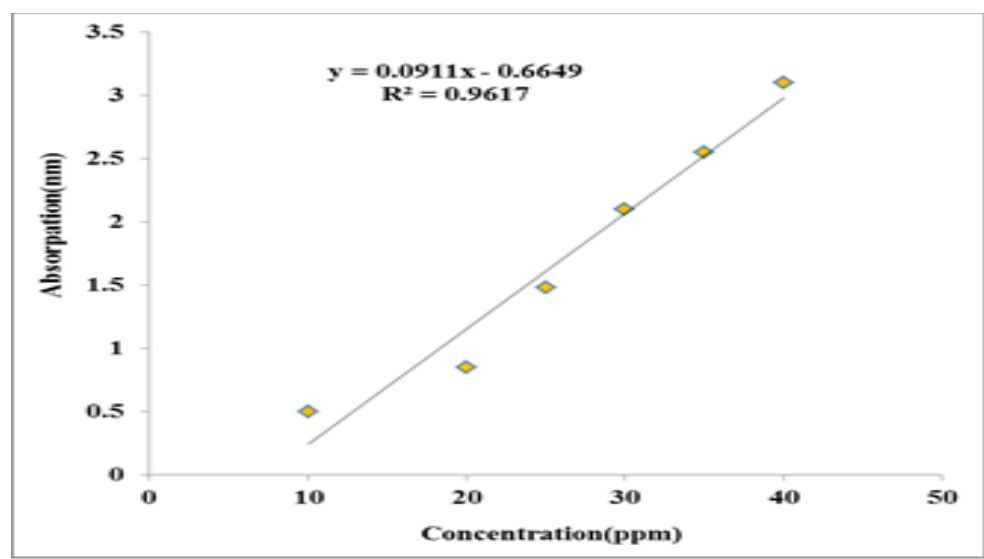

Figure 1: Calibration curve of bromo cresol purple after adsorption 


\subsubsection{Calculations of The Capacity of Adsorption.}

The amount of dye adsorbed per gm (qe) was calculated based on the following equation:

$$
\left(q_{\mathrm{e}}\right)=\frac{\left(c_{\mathrm{o}}-c_{\mathrm{e}}\right)}{m} \times v
$$

$\mathbf{C}_{\mathbf{o}}$ and $\mathbf{C}_{\mathbf{e}}$ are the initial and equilibrium concentration of adsorbate (here, bromo cresol purpule dye); $\mathbf{V}$ is the volume of dye solution (in liter); $\mathbf{m}$ is weight of adsorbent. The removal percentage of dye was calculated based on the following equation:

$$
\text { Removal } \%=\frac{\boldsymbol{c}_{\mathrm{o}}-\boldsymbol{c}_{\mathrm{e}}}{\boldsymbol{c}_{\mathrm{o}}} \times 100
$$

\subsubsection{Adsorption Isotherms}

Adsorption isotherms can be generated based on theoretical principles. Two isotherm equations have been tested in the present research, namely, Langmuir and Freundlich in order to describe the equilibrium characteristics of adsorption.

\subsubsection{Langmuir Adsorption Isotherm}

The most widely used isotherm equation for modeling the equilibrium is the Langmuir equation (9). The Langmuir linear equation is commonly expressed as followed:

$$
\frac{\mathrm{Ce}}{\mathbf{q e}}=\frac{\mathbf{1}}{\mathbf{k l}}+\left(\frac{\mathbf{a l}}{\mathbf{k l}}\right) \mathrm{Ce}
$$

A plot of Ce versus Ce/qe was linear showing the applicability of Langmuir adsorption isotherm for bromo cresol purple adsorption. $\mathrm{K}_{\mathrm{L}}$ and $\mathrm{a}_{\mathrm{L}}$ are the Langmuir constants related to adsorption capacity and rate of adsorption, respectively which are calculated from slope and intercept of the plot $\mathrm{Ce}$ versus $C \mathrm{e} / q \mathrm{e}$. The essential characteristics of Langmuir adsorption isotherm can be expressed in terms of a dimensionless constant, separation factor or equilibrium parameter 'RL' which is defined by:

$$
\mathrm{R}=\frac{1}{1+a l \cdot C i}
$$

Where, $\mathrm{Ci}=$ initial concentration of the dye and al=Langmuir constant. RL > 1 Unfavorable, RL $=1$ Linear, $0<\mathrm{RL}<1$ Favorable, RL=0 Irreversible.

\subsubsection{Freundlich Adsorption Isotherm}

The Freundlich isotherm model is the earliest known equation describing the adsorption process. It is an empirical equation and can be used for non-ideal sorption that involves heterogeneous adsorption. It also assumes that the adsorbent has a heterogeneous surface composed of adsorption 
sites with different adsorption potentials. This equation assumes that each class of adsorption site adsorbs molecules, as in the Langmuir equation. It is given by the following nonlinear equation below:

$$
\mathbf{q}=\mathbf{K C}
$$

where $\mathrm{KC}$ is a constant for the system, related to the bonding energy. $\mathrm{KC}$ can be defined as the adsorption or distribution coefficient and represents the quantity of dye adsorbed onto adsorbent for unit equilibrium concentration. $1 / \mathrm{n}$ is indicating the adsorption intensity of dye onto the adsorbent or surface heterogeneity, becoming more heterogeneous as its value gets closer to zero. A value for $1 / \mathrm{n}$ below 1 indicates a normal Freundlich isotherm while $1 / \mathrm{n}$ above 1 is indicative of cooperative adsorption (10). The above equation can be linearized in the logarithmic form the following equation and the Freundlich constants can be determined:

$$
\log q \mathrm{e}=\log \mathrm{K}_{\mathrm{F}}+\frac{1}{\mathrm{n}} \log \mathrm{Ce}
$$

A plot of $\log \mathrm{Ce}$ versus $\log$ qe was linear, where $\mathrm{K}_{\mathrm{F}}$ is measure of adsorption capacity (mg/g) and $\mathrm{n}$ is adsorption intensity. $1 / \mathrm{n}$ values indicate the type of isotherm to be irreversible $(1 / \mathrm{n}=0)$, favorable $(0<1 / \mathrm{n}<1)$, unfavorable $(1 / \mathrm{n}>1)$. The values of $1 / \mathrm{n}$ and $\mathrm{K}_{\mathrm{F}}$ can be calculated from the slope and intercept respectively.

\section{Results and Discussions}

\subsection{Adsorption Isotherms (Langmuir and Frindulich):}

\subsubsection{Langmuir Isotherms}

The effect of different times of adsorbent on the adsorption of the studied dyes was monitored. A linearized of Ce verus $\mathrm{C}_{\mathrm{e}} / \mathrm{q}_{\mathrm{e}}$ was obtained as shown in Table (1) and Figure (2).

Table 1: The values of langmuir adsorption isotherms for bromo cresol purple.

\begin{tabular}{|c|c|c|c|}
\hline $\mathbf{C}_{\mathrm{e}} / \mathbf{q}_{\mathbf{e}}$ & $\mathbf{q}_{\mathbf{e}}(\mathbf{m g} / \mathbf{g})$ & $\begin{array}{c}\text { Final concentration } \\
\mathbf{C}_{\mathbf{e}}(\mathbf{p p m})\end{array}$ & Time (min) \\
\hline 16.4893 & 0.5264 & 8.68 & 10 \\
\hline 16.5905 & 0.5256 & 8.72 & 20 \\
\hline 5.7857 & 0.6274 & 3.63 & 30 \\
\hline 3.1591 & 0.6584 & 2.08 & 40 \\
\hline 1.8090 & 0.6744 & 1.22 & 50 \\
\hline
\end{tabular}




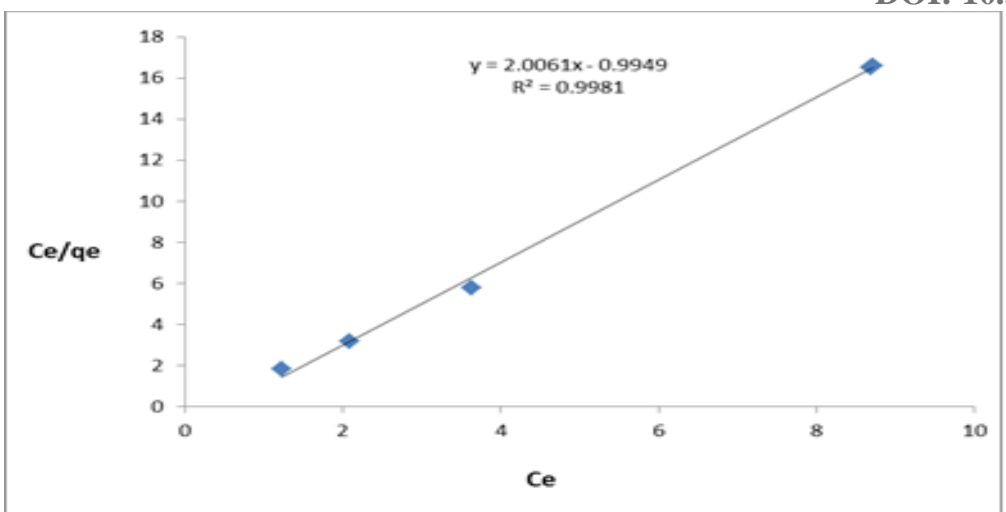

Figure 2: Langmuir adsorption isotherm for bromo cresol purple.

The fits are guite for the sorbent to suggest the application of the Langmuir model for the investigated system. The values of Langmuir parameter with correlation were computed from the intercept and the slop of the fitted Langmuir equation, Table (2).

Table 2: The Langmuir parameters for the adsorption bromo cresol purple using sea grasses.

\begin{tabular}{|l|c|c|c|c|}
\hline dye & $\mathbf{q}_{\max }$ & $\mathbf{a L}$ & $\mathbf{K l}$ & $\mathbf{r}^{\mathbf{2}}$ \\
\hline Bromo cresol purple & 0.5 & 2.018 & 1.006 & 0.998 \\
\hline
\end{tabular}

It seems the both values of $r^{2}$ of the studied dyes are high to assign the successful application to adsorbent the dye on the sea grasses. In the present study the values of RL were found to be less than (1) and slightly high than $(0)$ value $\approx 0.014$ indicating the favorable adsorption of the selected dyes on the sea grasses.

\subsubsection{Freundlich Adsorption Isotherm}

The values of $1 / \mathrm{n}$ and $\mathrm{K}_{\mathrm{F}}$ can be calculated from the slope and intercept respectively as shown in Table (3).

Table 3: Freundlich isotherms for the adsorption of bromo cresol purple.

\begin{tabular}{|l|l|l|l|}
\hline dye & $\mathbf{1} / \mathbf{n}$ & $\mathbf{K F}$ & $\mathbf{r}^{\mathbf{2}}$ \\
\hline Bromo cresol purple & 0.138 & 0.71 & 0.99 \\
\hline
\end{tabular}

The results of Freundlich of the present study were illustrated in Table (4) and represented in Figure of (3).

Table 4: The values of Fruendlich adsorption isotherms for bromo cresol purple adsorption.

\begin{tabular}{|c|c|c|c|c|}
\hline $\begin{array}{c}\text { Time } \\
(\mathbf{m i n})\end{array}$ & Final Concentration $\mathbf{C}_{\mathbf{e}}$ & $\log \mathbf{C e}$ & $\mathbf{q}_{\mathbf{e}}(\mathbf{m g} / \mathbf{g})$ & Log qe \\
\hline 10 & 8.68 & 0.9385 & 0.5264 & -0.2786 \\
\hline 20 & 8.72 & 0.9405 & 0.5256 & -0.2793 \\
\hline 30 & 3.63 & 0.560 & 0.6274 & -0.2024 \\
\hline 40 & 2.08 & 0.320 & 0.6584 & -0.1815 \\
\hline 50 & 1.28 & 0.110 & 0.6744 & -0.1710 \\
\hline
\end{tabular}




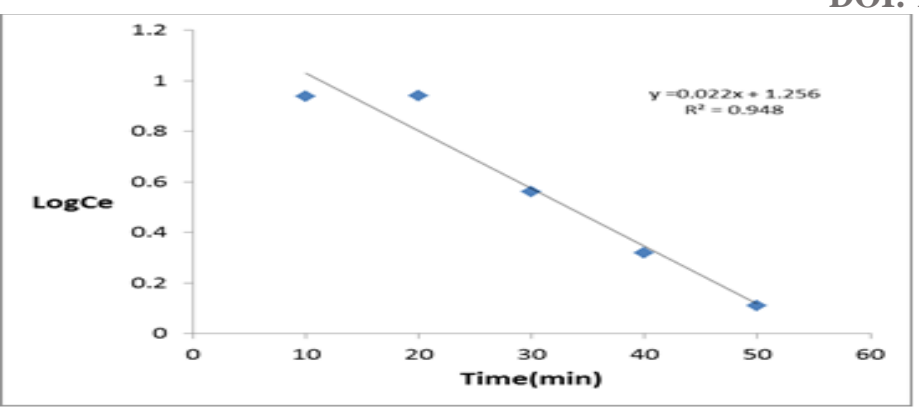

Figure 5: The relation between Time ( $\min )$ and $\log C_{e}$ of the bromo cresol purple.

\section{Conclusions and Recommendations}

According to the results which recorded in this manuscript the activated carbon which prepared from low cost material (sea grasses) showed high capacity to removal the studied dye, also the adsorption behavior gave very interesting isotherms of Langmuir and frendlish parameters.

\section{Acknowledgements}

The authors gratefully thank to Faculty of Science and Technology, Omar Al-Mukhtar University, Al Bayda (OMU) for providing the facilities for the research work.

\section{References}

[1] Weber, Jr. W. Rates of adsorption In: Physicochemical Processes for Water Quality Control. New York, Wiley- Inter science; 1972.

[2] Hasar, H. adsorption of nickel (II) from aqueous solution onto activated carbon prepared from almond husk, J. hazard. Mater; 2005,97

[3] Patil, S. Renukdas and N. Patel. Removal of methylene blue, a basic dye from aqueous solutions by adsorption using teak tree ( Tectonagrandis) bark powder. International J. of Environmental Sciences ;2011, 1,711-726

[4] El-Zahhar,A.A., N.S.Awwad and E.E. El-Katori. Removal of bromophenol blue dye from industrial wastewater by synthesizing polymer-clay composite. J. of Molecular Liquids; 2014,199,454-461.

[5] Pavan, F.A., A. C.Mazzocato and Y.Gushikem. Removal of methylene blue dye from aqueous solutions by adsorption using yellow passion fruit peel as adsorbent. Bio resource Technology; 2008,99, 3162-3165.

[6] Boehnke, D.N., and R. del Delumyea. Determination of the octanol/water partition coefficient for organic pollutants of varying Hydrophilic character; 1999,173-178.

[7] Rahman,M.A., S. M. R. Amin and A. M. S.Alam. Removal of Methylene Blue from wastewater using activated carbon prepared from rice husk. Dhaka Univ. J. Sci; 2012, (60)2,185-189.

[8] Jain,A.K., V.K. Gupta, A. Bhatnagar and Suhas, M.N. Utilization of industrial waste products as adsorbents for the removal of dyes. J. of Hazardous Materials B101; 2003, 31-42.

[9] Langmuir 1916, The constitution and fundamental properties of solids and liquids .J.Am.Chem.Soc., 38:2221-2295.

[10] Freundlich, H.M.F.Z,1906.Over the adsorption in solution ,j.phys.Chem;57:385-470

*Corresponding author.

E-mail address: enas.almadani@ omu.edu.ly 